\title{
Revisión de las primeras experiencias de resinación en Galicia (1950-1970)
}

Recibido: 15 febreiro 2016 / Aceptado: 19 outubro 2016

(c) IBADER- Universidade de Santiago de Compostela 2016
Resumen En este artículo, se revisan las experiencias de resinación desarrolladas en Galicia desde la década de los 50 a los 70 , basándose en publicaciones y en el estudio de antiguos documentos rescatados del Instituto Forestal de Investigaciones y Experiencias (IFIE). Los documentos recopilados resumen los ensayos de investigación realizados en Galicia que en primer lugar, pueden servir de base para facilitar las futuras investigaciones sobre resinación que se realicen en el noroeste de la Península Ibérica, y en segundo lugar, aportar unos primeros fundamentos que permitan desarrollar una selvicultura que compatibilice la extracción complementaria de resina con el aprovechamiento de la madera en el pino pinaster en Galicia.

Palabras clave Galicia, pica de corteza, Pinus pinaster Ait., selvicultura, sistema de resinación.

First experiences in resin production between the ' $50 \mathrm{~s}$ and the ' 70 s in the northwestern Spansh region of Galicia

Abstract In this article, first experiences in resin production between the ' 50 s and the '70s in the northwestern Spanish region of Galicia are reviewed. This report is primarily based on the study of publications and old documents retrieved from the IFIE -the Spanish Forestry Institute of Research and Experience, which outline the researches conducted in Galicia. The review of these articles can, on the one hand, facilitate future researches that could be carried out in the north-west of the Iberian Peninsula. On the other hand, it may also provide the initial basis for the development of a silviculture that makes supplementary resin tapping compatible with the logging of maritime pine in Galicia.

Enrique Martínez Chamorro

Centro de Investigación Forestal de Lourizán. Consellería do

Medio Rural, Xunta de Galicia Ctra. Pontevedra-Marín Iglesia, 19

Lourizán 36153 Pontevedra.

Tfno.: 986805000 Fax: 986805031

E-mail: enrique.martinez.chamorro@xunta.es
Keywords Bark Streak Method, North-West of the Iberian Peninsula, Pinus pinaster Ait., Resin Tapping, Silviculture.

\section{Introducción}

El aprovechamiento resinero consiste en la realización de incisiones en el tronco de los árboles, que permiten la recogida de la miera, materia prima empleada en la industria resinera y que está constituida por una mezcla de sustancias volátiles (aguarrás), y un cuerpo sólido de aspecto vítreo (no volátil) que se obtiene como condensado en la destilación de la miera purificada o trementina (colofonia). El aprovechamiento de resina o miera en España alcanzó su máxima expansión a principios de los años 60 del siglo XX, en el que se totalizaron 270000 ha resinadas (Serrada, 2004), alcanzando, su máxima producción en el año 1961, con más de 55000 toneladas, y en Portugal en el año 1973, superando las 90000 toneladas (Picardo, 2013).

El Pinus pinaster es la especie española del género Pinus que mejor se adapta al aprovechamiento resinero, aunque se han resinado también otras especies. Los pinares de mayor producción resinera han sido los de la Meseta norte, en las provincias de Segovia, Ávila o Valladolid, si bien el aprovechamiento afectó también a pinares de serranía en Soria, Cuenca, León, Guadalajara Albacete, Teruel y Málaga, aunque con producciones más modestas (Rodríguez et al., 2008). Los sistemas de resinación fueron evolucionando durante el siglo $\mathrm{XX}$, desde los que afectaban a la madera (albura) hasta los que hicieron compatible el aprovechamiento resinero del pino con su explotación maderera.

No obstante, la década de los ochenta marca el inicio de una crisis en las explotaciones resineras, tanto en España como Portugal, debido fundamentalmente, a la pérdida de la cuota de mercado, provocada por los elevados costes de producción, la disminución de la mano de obra, y sobre todo, la entrada de China en el mercado con precios muy inferiores a los europeos (Anastácio \& Carvalho, 2008). Sin 
embargo, China actualmente ha bajado el ritmo de sus exportaciones, obligada por la caída de la producción nacional, motivada por la pérdida de mano de obra en el campo, el incremento de los costes salariales y a las malas prácticas y planificación de sus aprovechamientos resineros que han deteriorado, en muchos casos, su patrimonio forestal (Picardo, 2013).

Teniendo en cuenta este contexto, el resurgir de este aprovechamiento en el norte de Portugal (Santos, 2013) y en Castilla y León y las perspectivas generadas en Galicia, el objetivo de este artículo es revisar los primeros ensayos sobre sistemas de resinación realizados en Galicia, recopilando los resultados de sus producciones, definiendo los métodos y técnicas de resinación empleados, y analizando cuales de las conclusiones obtenidas, pueden tener interés a la hora de resinar estos pinares atlánticos.

\section{Los métodos de resinación ensayados en Galicia}

Desde 1940 hasta 1970, se iniciaron en la Península Ibérica ensayos y experiencias de resinación con diferentes sistemas (Radich, 1995; Hernández, 2006). En España, fue el Instituto Forestal de Investigaciones y Experiencias (IFIE), a través de su sección de resinas y productos derivados, el que promovió varias pruebas con diferentes sistemas de resinación.

A comienzos de los años 50, el antiguo IFIE, en colaboración con los servicios forestales provinciales y, especialmente, con el Distrito Forestal de Pontevedra, realizó experiencias sobre resinación en Galicia que se prolongaron hasta principios de los años 70 . Galicia, pese a no ser una región tradicionalmente resinera, captó enseguida el interés de industriales y de la propia administración, debido a las extensas masas de Pinus pinaster Ait. existentes y a la necesidad de impulsar la producción de resina fuera de las regiones tradicionalmente resineras del centro peninsular.

De los ensayos y experiencias que se muestra en este artículo, se deduce que en Galicia se emplearon al menos 4 sistemas de resinación:

el sistema Hugues, tradicionalmente aplicado desde el s. $\mathrm{XIX}$, en el que se realizaba una incisión o herida vertical y alargada en la superficie descortezada del tronco, consistente en arrancar de arriba hacia abajo unas cuantas astillas o virutas hasta penetrar en la albura 1,5 $\mathrm{cm}$ de profundidad máxima, dejando en la superficie del tronco una cara cóncava con pérdida de madera (Calderón, 2009; Hernández, 2006); (Fotografía 1.a)

el sistema Mazek de "espina de pescado", consiste en realizar, con un cepillo del mismo nombre, una serie de estrías largas y trasversales o canales en forma de $\mathrm{V}$, incisiones que forman un ángulo de $45^{\circ}$ con el eje del árbol y que permiten que la miera se deslice facilitando así su recogida (Nájera et al.,1957; Solís, 1968); (Fotografía 1.b) el sistema de caras múltiples, donde se realizaban picas ${ }^{1}$ largas y continuas de $10 \mathrm{~mm}$ de ancho a lo largo del eje del árbol, y en número de 5. Aquí, la cicatrización es muy rápida, así que se pueden volver a explotar las caras que se reconstituyen (Calderón, 2009); (Fotografía 1.c)

el sistema americano de "pica de corteza", consistente en la realización de las picas o incisiones aproximadamente horizontales con una azuela o escoda y la aplicación de un estimulante ácido, siempre previo descortezado del pino (Pinillos et al., 2004); (Fotografía 1.d)

En 1952, el IFIE inició en Coca (Segovia) experiencias con el, por entonces novedoso, sistema de resinación de pica de corteza, que consistió, en primer lugar, en la adaptación del sistema y comprobación de sus rendimientos en producción de miera. A ello, siguió una segunda fase de extensión del ensayo a todo el ámbito nacional, comparando el nuevo sistema de pica de corteza con estimulación y sus variantes con el sistema Hugues, sobre más de 200000 pinos de
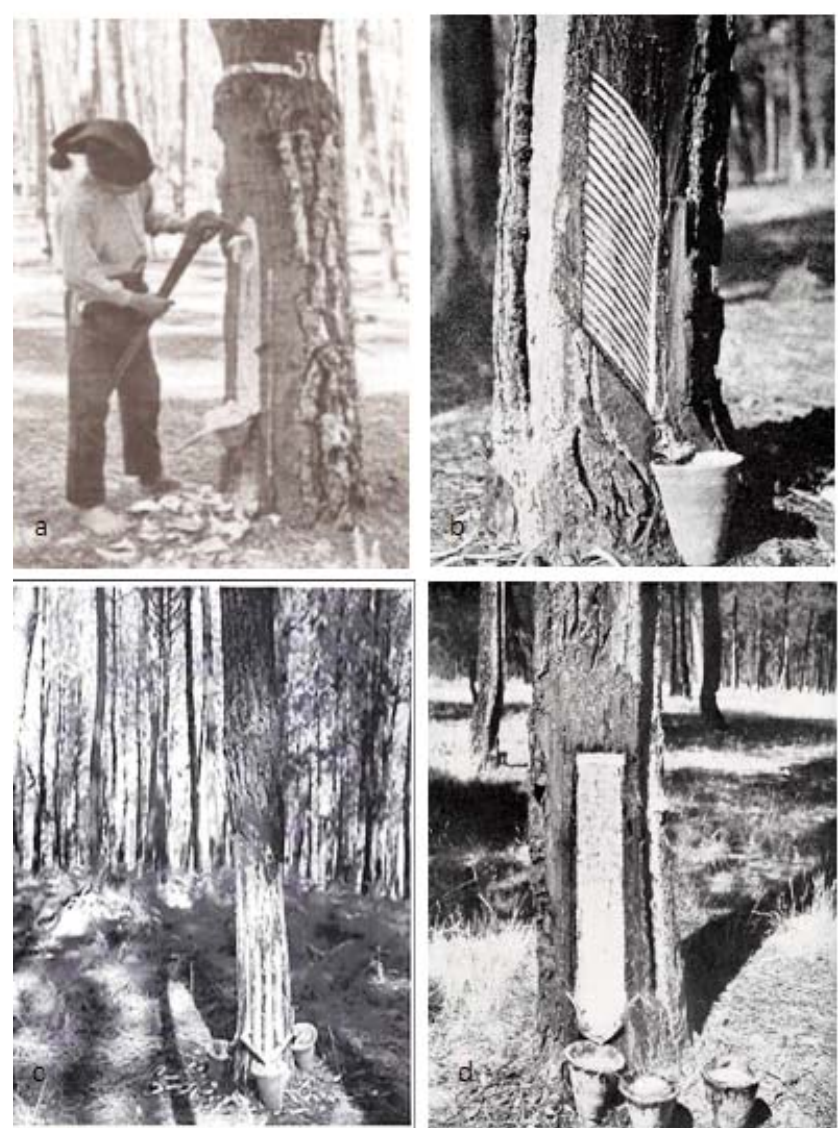

Fotografía 1.- a. Resinación no Pinhal do Rei. Marinha Grande (Portugal) por el método Hugues o francés. Fuente: A exposição "Factos e Personalidades do Pinhal do Rei".2011, b. Resinación por el sistema Mazek el Alijar. Las Navas del Marqués (Ávila) Fuente: fototeca forestal. INIA, c. Parcela de investigación. Ensayo de extracción por el método español de "caras múltiples". Localización probable: península del Morrazo (Pontevedra). Fuente: biblioteca CIF de Lourizán, d. Sistema americano de resinación de Pica de Corteza; entalladura estimulada con ácido sulfúrico. Fuente: fototeca forestal INIA 
distintas especies, de las que destacan: Pinus pinaster Ait., Pinus halepensis Mill. y Pinus nigra Arnold, constantando una mejora de los tiempos de extracción, una mayor facilidad de aprendizaje y un incremento de producción de miera de más del 10\% (IFIE, 1965).

\section{Experiencias de los años 50: ensayo de métodos y producciones}

Las primeras experiencias de las que se tiene constancia en Galicia son de los años 1952-1953 y consistieron en probar el sistema Mazek, que tenía como principal ventaja su menor consumo de madera y su mayor facilidad de aprendizaje, a pesar de ser menos productivo que el sistema Hugues (Nájera et al., 1957). Se escogieron, en la península del Morrazo, dos rodales destinados a ser cortados al terminar las dos campañas de resinación que se proponían, motivo por el que se abrieron todas las caras ${ }^{2}$ que el diámetro de los árboles $(23 \mathrm{~cm}$ de media) permitía. Se consideraron dos factores: el número de entalladuras ${ }^{3}$ a abrir (2 o 3) y la estimulación química, con y sin aplicación de $\mathrm{H}_{2} \mathrm{SO}_{4}$ al $50 \%$.

Se abrieron, por tanto, dos o tres entalladuras sobre otras tantas caras de $12 \mathrm{~cm}$ de anchura, tomando como testigo árboles resinados con el método Hugues, también con dos o tres entalladuras. En el caso de la resinación Mazek, se ensayó la estimulación química con $\mathrm{H}_{2} \mathrm{SO}_{4}$ al $50 \%$, con los resultados que se muestran en la Tabla 1 , donde se aprecia una reducción de producción del 37 al 50\% en Mazek respecto a Hugues cuando no se aplica estimulación, si bien las reducciones en producción son menores cuando el sistema Mazek se combina con estimulación (del 10 al 44\% inferior). La apertura simultánea de tres entalladuras supone que el efecto de la estimulación química resulta menos importante. Estos resultados, según los autores mencionados anteriormente, se deben a la falta de conocimientos de la mano de obra y al escaso diámetro de los árboles.

La segunda experiencia se realizó con caras de igual anchura que el diámetro del pino, empleando el sistema Mazek sin estimulación y con estimulación de $\mathrm{H}_{2} \mathrm{SO}_{4}$, pero, en este caso, con concentraciones al $30 \%$, al $40 \%$ y al $50 \%$; en la Tabla 2, aparte de observarse resultados similares a los de la Tabla 1, no se constatan tampoco diferencias de producción entre los porcentajes de ácido sulfúrico empleados. Igual que en el caso anterior, los autores ponen de manifiesto que los diámetros normales y la pericia de los resineros eran bajos.
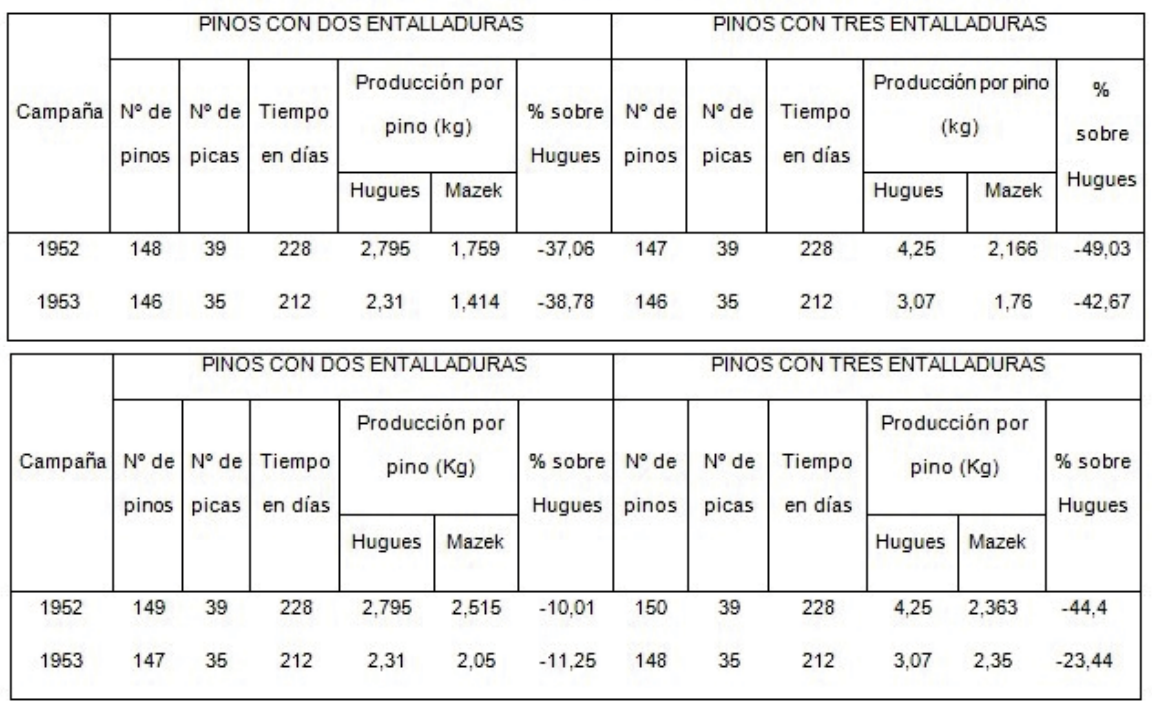

Tabla 1.- Sistema MAZEK normal con entalladura de $12 \mathrm{~cm}$ (Arriba) y Sistema MAZEK con $\mathrm{SO} 4 \mathrm{H} 2$ al $50 \%$ y entalladura de $12 \mathrm{~cm}$ (Abajo). Sitio de ensayos de O Morrazo (Pontevedra)

\begin{tabular}{|c|c|c|c|c|c|c|c|c|c|c|c|c|c|c|c|}
\hline \multirow[b]{2}{*}{ ENSAYO } & \multicolumn{5}{|c|}{1953} & \multicolumn{5}{|c|}{1954} & \multicolumn{5}{|c|}{1955} \\
\hline & $\begin{array}{l}N^{\circ} \text { de } \\
\text { pinos }\end{array}$ & $\begin{array}{l}N^{\circ} \text { de } \\
\text { picas }\end{array}$ & dias & $\begin{array}{c}\text { Prod. } \\
\mathrm{kg}\end{array}$ & $\begin{array}{l}\% \text { respecto } \\
\text { Hugues }\end{array}$ & $\begin{array}{l}N^{\circ} \mathrm{de} \\
\text { pinos }\end{array}$ & $\begin{array}{l}N^{\circ} \text { de } \\
\text { picas }\end{array}$ & dias & $\begin{array}{c}\text { Prod. } \\
\mathrm{kg}\end{array}$ & $\begin{array}{l}\% \text { respecto } \\
\text { Hugues }\end{array}$ & $\begin{array}{c}\mathrm{N}^{\circ} \\
\mathrm{de} \\
\text { pinos }\end{array}$ & $\begin{array}{l}N^{\circ} \text { de } \\
\text { picas }\end{array}$ & dias & $\begin{array}{l}\text { Prod } \\
\mathrm{kg} .\end{array}$ & $\begin{array}{l}\text { \% respecto } \\
\text { Hugues }\end{array}$ \\
\hline $\begin{array}{l}\text { Sistema } \\
\text { Hugues }\end{array}$ & 100 & 34 & 172 & 2,31 & & 100 & 36 & 210 & 2,908 & & 100 & 39 & 230 & 3,934 & \\
\hline MAZEK normal & 50 & 34 & 172 & 1,455 & $-37,1$ & 49 & 36 & 210 & 2,067 & $-28,98$ & 44 & 39 & 230 & 2,198 & $-44,12$ \\
\hline \multicolumn{16}{|l|}{$\begin{array}{c}\text { MAZEK } \\
\text { estimulado }\end{array}$} \\
\hline $\mathrm{SO}_{4} \mathrm{H}_{2}$ al $30 \%$ & 50 & 34 & 172 & 1,905 & $-17,53$ & 49 & 36 & 210 & 2,415 & $-17,33$ & 43 & 39 & 230 & 2,458 & $-37,52$ \\
\hline $\mathrm{SO}_{4} \mathrm{H}_{2}$ al $40 \%$ & 50 & 34 & 172 & 2,075 & $-10,21$ & 49 & 36 & 210 & 2,538 & $-12,72$ & 43 & 39 & 230 & 2,337 & $-39,57$ \\
\hline $\mathrm{SO}_{4} \mathrm{H}_{2}$ al $50 \%$ & 50 & 34 & 172 & 1,94 & $-16,01$ & 49 & 36 & 210 & 2,72 & $-6,46$ & 43 & 39 & 230 & 2,62 & $-33,4$ \\
\hline
\end{tabular}

Tabla 2.- Resinación intensiva: Sistema MAZEK con entalladura a su diámetro: HUGUES dos entalladuras. Sitio de ensayo de O Morrazo (Pontevedra) 
Dentro de esta fase de extensión del ensayo propuesta por la sección de resinas, probablemente en el año 1954-55, se produjeron las segundas experiencias de resinación en Galicia, también en la península del Morrazo (Pontevedra). Se trataba de probar distintos sistemas de resinación, con y sin estimulación química, abriendo tres clases de caras de resinación: de $12 \mathrm{~cm}$, de anchura igual al diámetro normal del pino y abriendo caras en todo el árbol (Nájera, 1956). Se emplearon, aparte del anterior sistema, 2 nuevos sistemas de resinación:

el sistema americano de "pica de corteza", empleando resinación normal y estimulada con $\mathrm{H}_{2} \mathrm{SO}_{4}$ en concentraciones de $30-40-50 \%$, en las tres clases descritas, sistema que también se aplicó a Pinus radiata D. Don con ácido al $40 \%$.

el sistema "caras múltiples", aplicado a Pinus pinaster Ait. y Pinus radiata D. Don con una o dos caras de $12 \mathrm{~cm}$ para el primero y una sola de $12 \mathrm{~cm}$. para el segundo.

En esta segunda experiencia, volvió a quedar limitada por la delgadez de la masa, aunque sí se concluyó que el rendimiento fue superior al del sistema Hugues en los casos en los que se empleó $\mathrm{H}_{2} \mathrm{SO}_{4}$ como estimulante.

En el año 1957, dentro de esta misma experiencia, el ingeniero jefe de la sección de resinas y productos derivados del IFIE realiza un documento titulado: "Informe sobre la posible resinación de los pinares de Galicia y estudio de las bases generales que deberá cumplir este aprovechamiento", con firma ilegible. Aparte de reflejar la experiencia anterior, aporta datos sobre la época de resinación:

“[...] La campaña de resinación se iniciaba en el mes de marzo con las labores de preparación, consistentes en la realización de descortezado (desroñe), la colocación de la grapa ${ }^{4}$ que conduce la miera hasta el pote, sujeta al fuste con la ayuda de una punta. Durante los meses de abril a septiembre, se llevaba a cabo la resinación propiamente dicha con la ejecución de las picas, las recolecciones periódicas o remasas y el almacén de la miera en barricas. La campaña finaliza en octubre con la recogida del barrasco ${ }^{5}$, que es la miera adherida en la entalladura, y la última remasa, retirando, finalmente, potes, puntas y grapas [...]".

Durante esta segunda experiencia, ampliada ahora en el tiempo (1957) y en el espacio, se emplearon en Galicia los tres sistemas de resinación comentados, así como el sistema Hugues como testigo, y se concluyó que el que reunía mejores condiciones para el pino pinaster de Galicia era el citado sistema americano de pica de corteza estimulado con $\mathrm{H}_{2} \mathrm{SO}_{4}$ al $30-40 \%$.

Resulta relevante también el informe de 1957 (Nájera, 1957), en el que se constata una ampliación de la experiencia a diferentes localizaciones del sur de Galicia: Tomiño, Ponteareas y Entrimo (Fotografía 2.a) y en el que, aun descartando el sistema Hugues, se hace un estudio de producción comparada con este sistema, utilizando árboles de más de $30 \mathrm{~cm}$ de diámetro normal y obteniendo una media de $2,76 \mathrm{~kg}$ de miera por pie abierto y campaña durante los cuatro años de experiencias. También existe constancia de una segunda experiencia de la Diputación de Pontevedra en pinares repoblados en la península del Morrazo (Pontevedra) con diámetros entre los 22 y $23 \mathrm{~cm}$ y donde la producción de miera por el método Hugues en caras de $12 \mathrm{~cm}$ fue de $2,25 \mathrm{~kg}$ de media.

En la Figura 1, se muestran la media de los resultados obtenidos durante 4 años con este sistema en sitios de ensayo con resinaciones a vida 6 y a muerte ${ }^{7}$ con dos dimensiones de cara; un hecho destacable es el establecimiento de caras estrechas de $9 \mathrm{~cm}$ de anchura con el método Hugues, de gran importancia en Galicia, para que la madera se depreciara lo menos posible.

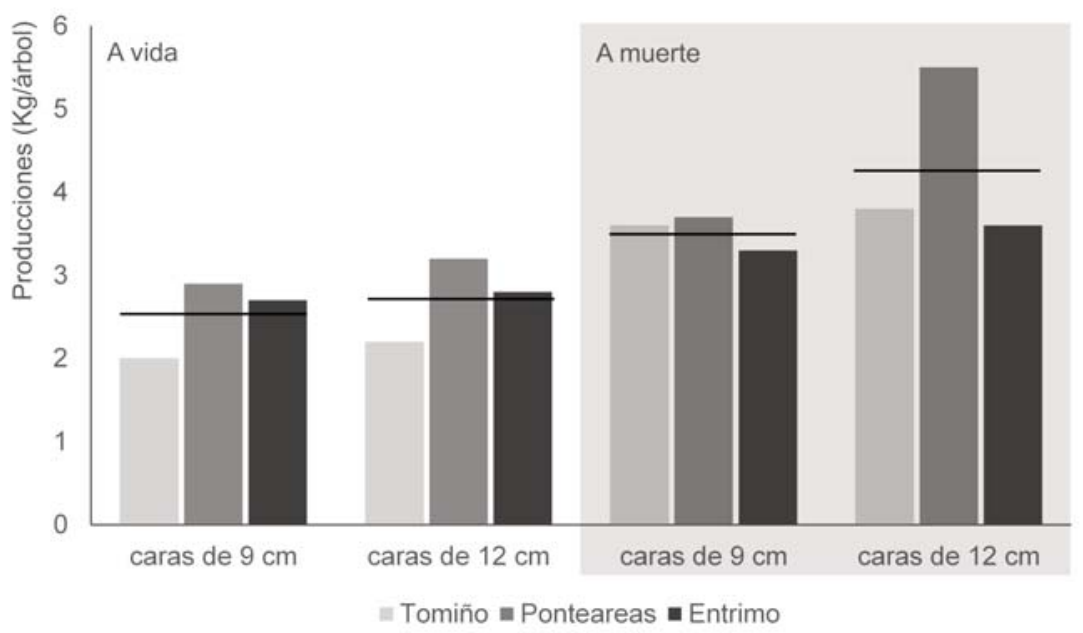

Figura 1.- Producciones (kg/árbol) en a las experiencias realizadas en Galicia en la década de los 50 con el método Hugues

4 Grapa: placa fina de zinc o hierro galvanizado que sirve para recoger la resina que escurre por la cara para llevarla al pote.

5 Barrasco: conjunto de resina que se recoge al rascar la entalladura al final de la campaña y que va unida a virutas, cortezas, insectos, etc. También es una herramienta para desroñar o quitar la corteza del pino que se va a picar.

6 Resinación a vida: se mantiene abierta una única cara en cada momento.

7 Resinación a muerte: se le abren simultáneamente todas las caras posibles. 
Analizando estos datos de producción en vida con caras de $12 \mathrm{~cm}$, el informe deduce que son valores prácticamente iguales a los valores medios españoles en resinación, que oscilan entre los $1,9 \mathrm{~kg}$ de miera por pie abierto y campaña de Teruel y Málaga y los 4 kg de Segovia.

Una vez que concluye que el sistema de pica de corteza con estimulación, sin duda, dará una producción superior a la del sistema Hugues, el informe establece dos requisitos o condiciones que considera básicas y que debe cumplir el método de resinación que se aplique en Galicia:

ser compatible con el actual aprovechamiento maderable, en el sentido de no perturbar el volumen de madera que, anualmente, se corta.

que su manejo sea cómodo y de aprendizaje fácil y rápido.

Continúa el informe indicando que la miera gallega tiene una cantidad de agua e impurezas sólidas superior a la del resto de regiones resineras, lo que rebajará la pureza de colofonia ${ }^{8}$ obtenida.

Por otra parte, después de analizar los datos estudiados, el autor propone una selvicultura en donde la resinación sea un aprovechamiento completamente secundario del maderable y esté basada en la apertura de dos entalladuras opuestas en los dos años anteriores a su aprovechamiento maderable, en masas próximas a su turno de corta, fundamentando este proceder en las siguientes razones: la poca importancia de los crecimientos perdidos y la comodidad del trabajo para el resinero (concentración del trabajo).

Finalmente, pronostica una primera estimación de la producción de miera, ayudándose de las estadísticas forestales de cortas de 1953-1954, concluyendo que el pino medio aprovechado para madera en Galicia pertenece a la clase diamétrica 25 y que las existencias susceptibles de ser resinadas rondan los 3,3 millones de pinos. Teniendo en cuenta estos datos y la propuesta de resinar durante los dos años anteriores a su corta con dos caras opuestas, y admitiendo por año una producción de miera de $3,5 \mathrm{~kg} /$ pino, realiza el siguiente cálculo sobre la producción teórica posible de miera anual en Galicia:

\section{3,3 M de pinos $x 2$ años $\times 3,5 \mathrm{~kg} / \mathrm{años}=23100 \mathrm{~T}$}

Lo que supondría alrededor del $50 \%$ de la producción nacional, que era de $43000 \mathrm{~T}$.

\section{Experiencias de los años 60: condiciones del aprovechamiento y compatibilidad con la madera}

Otro informe elaborado por la Sección de resinas en el año 1961 y titulado: "Informe sobre la resinación de los pinares de Galicia y condiciones generales que se proponen para la concesión de este aprovechamiento", cuyo autor es Fernando Nájera y Angulo, con la colaboración de Rafael Boulet Sirvent, analiza desde las condiciones que debe reunir la resinación en Galicia hasta las bases para la concesión del aprovechamiento de los pinares de Galicia, estableciendo las directrices de un plan quinquenal (1963/67).

Del informe, cabe destacar que matiza las dos condiciones, ya mencionadas en el informe de 1957, en cuanto a las condiciones que deben reunir el sistema de resinación que se utilice en Galicia, e incluye otras nuevas:

la resinación debe ser un aprovechamiento secundario frente a la madera, por lo que debe propugnarse que el periodo de resinación abarque, como máximo, los cinco años inmediatamente anteriores a la corta del pino.

se deberá realizar una cara de resinación que no afecte apenas al crecimiento en diámetro ni al rendimiento en madera aserrada.

el minifundismo de la propiedad forestal hace imposible la resinación con obreros contratados para trabajar un determinado número de pinos, por lo que solo cabe la resinación de fincas o rodales próximos a ser cortados que se correspondan con zonas de fácil y rápido recorrido.

Especial interés tiene el informe a la hora de plantear soluciones en el contexto de Galicia tendentes a mejorar las cantidades de miera recogida. Según Nájera y Boulet, las cantidades recogidas son de un 20 a un $25 \%$ menores que las producidas debido al derrame de parte de la miera que se obtiene por la lluvia. Sobre este problema, se plantean soluciones que van desde el aumento de la capacidad de los potes en un $50 \%$ (potes con diámetros exteriores de 80 a $170 \mathrm{~mm}$ y alturas de $140 \mathrm{~mm}$ ) hasta el empleo de tapas. Ambas actuaciones permitirían, por un lado, retrasar el llenado del pote a consecuencia de las abundantes pluviometrías de Galicia, permitiendo a los resineros ganar tiempo para su vaciado y, por otro, con las tapas, evitar la evaporación del aguarrás y la caída de impurezas. Concluyen que, con estas mejoras en el sistema de recogida de la miera, aumentaría en más de un $10 \%$ el rendimiento por pino.

Como resultado final, los investigadores proponen como sistema óptimo para los pinares gallegos el de pica de corteza con estimulación a base de $\mathrm{H}_{2} \mathrm{SO}_{4}$ al $40-50 \%$.

Esta experiencia concluye que la cifra de $2 \mathrm{~kg}$ por entalladura podría servir de base, con carácter mínimo, para el cálculo de la capacidad resinera de los pinares en Galicia, compuestos mayoritariamente, en aquel entonces, por masas con una espesura excesiva con árboles de 20 a 25 $\mathrm{cm}$ en su mayoría.

El resto del informe se dedica a establecer las bases para aprovechamientos quinquenales de los pinares de Galicia: la posibilidad anual de producción de miera en función de las existencias, las medidas y las condiciones descritas, el análisis de la capacidad de consumo de productos resinosos en Galicia y las posibilidades de exportación de estos productos, analizando también la viabilidad de construir dos fábricas de destilación en Galicia. Concluyendo que, en tanto no se determinen las condiciones que debe reunir la resinación en Galicia por 
parte de la Dirección General de Montes, se desaconseja la construcción de fábricas de destilación.

Por otra parte, también se refleja la experiencia realizada por la Diputación de Pontevedra sobre 20000 pinos en los montes del Morrazo. Esta experiencia se cita en el: "Informe sobre las experiencias de producción de miera por el sistema de resinación de pica de corteza de 1962 de la Sección de resinas y derivados" (IFIE, 1962), donde se desprende que se continuaron 5 años más con los ensayos del sistema de pica de corteza con los resultados que se observan en la Tabla 3.

En la experiencia, se determina que el periodo de resinación anual fue de aproximadamente 7 meses, muy similar o incluso algo superior a los de las otras experiencias realizadas en el centro de la Península Ibérica; también se señala que el número de picas fue similar al resto y que las producciones fueron algo más bajas, toda vez que los ensayos se realizaron en periodos cortos y en árboles de diámetros pequeños $(20-25 \mathrm{~cm}$ de diámetro normal) que iban a ser cortados en el plazo de uno a tres años. El informe concluye: "[...] al final del año 1961 se suspendieron ya estos ensayos, por haber quedado suficientemente estudiada y probada la posibilidad de resinación en Galicia $[\ldots] "$

No obstante, la intención del IFIE con estas experiencias era obtener la máxima información posible acerca del sistema de resinación idóneo que debía implantarse en los pinares gallegos y que se estaba realizando de forma similar en otras parcelas del resto de España, sin estudiar los efectos de la pérdida de volumen.

García-Borregón (1966) ya indicaba la necesidad de conocer cuáles son las verdaderas pérdidas de crecimiento anual que la aplicación de la resinación produce en la madera de los pinos. Su preocupación es lógica toda vez que, según él mismo manifiesta, el precio medio de la madera en pie, en 1966, para árboles de 20 a $39 \mathrm{~cm}$ era de 800 ptas $/ \mathrm{m}^{3} \mathrm{cc} 9\left(4,808 € / \mathrm{m}^{3} \mathrm{cc}\right)$; valor que, actualizado, supondría $121,02 € / \mathrm{m}^{3} \mathrm{cc}$.

Muy probablemente, la importancia de la madera como recurso, junto a su precio, ya había sido puesta de manifiesto en anteriores informes, lo que hizo que el IFIE, en 1967, iniciara un "estudio relativo a la influencia que ejerce la resinación, por el sistema de pica de corteza con estimulación, en el crecimiento maderable" (IFIE, 1967), ante la falta de conocimientos sobre la influencia de este sistema en el crecimiento maderable y las características físicas, químicas y mecánicas de la madera en la industria del aserrado.

Con este fin, se eligió el monte Seixos Albos de Tui, al considerarse un lugar representativo de los pinares de Galicia con capacidad para ser resinados: diámetros normales de 20 a $40 \mathrm{~cm}$ y alta densidad. Durante el período de 1967 a 1971, se replanteó en este monte una parcela donde se resinaron, en principio, 100 pinos y se dejaron un número igual de pies testigos sin resinar, elegidos todos ellos por procedimientos estadísticos. Se realizó una

\begin{tabular}{|c|c|c|c|c|c|}
\hline Año & $\mathrm{N}^{0}$ de pinos & Dias & Picas & Entalladuras & Prod. Media.(kg) \\
\hline 1957 & 14.750 & 209 & 29 & $1^{2}$ & 2,040 \\
\hline \multirow{2}{*}{1958} & 14.750 & \multirow{2}{*}{216} & \multirow{2}{*}{29} & $2^{a}$ & \multirow{2}{*}{1,808} \\
\hline & 11.950 & & & $1^{a}$ & \\
\hline \multirow{2}{*}{1959} & 15.000 & \multirow{2}{*}{229} & \multirow{2}{*}{29} & $1^{2}$ & \multirow{2}{*}{1,931} \\
\hline & 11.000 & & & $2^{\mathrm{a}}$ & \\
\hline \multirow{2}{*}{1960} & 15.000 & \multirow{2}{*}{220} & \multirow{2}{*}{29} & $1^{2}$ & \multirow{2}{*}{1,916} \\
\hline & 11.000 & & & $3^{a}$ & \\
\hline 1961 & 22.000 & 217 & 28 & $1^{2}-2^{a}-3^{2}$ & 2,228 \\
\hline
\end{tabular}

Tabla 3.- Resumen de experiencias de producción de miera con el sistema de pica con corteza en el Morrazo (Pontevedra) 
cubicación inicial pie a pie, en el momento de instalar la parcela, para empezar a realizar la resinación por el sistema de pica de corteza con estimulante líquido y comparar las mediciones con otros 100 testigos a los 3 y a los 5 años, cumpliendo dos premisas:

El periodo de resinación debería ser los 5 años anteriores a la corta.

Los pinos deberán tener más de $20 \mathrm{~cm}$ de diámetro normal.

La experiencia se reforzó en 1969 añadiendo una segunda parcela con 54 pinos más, divididos en dos grupos de 27 , para conocer las posibilidades de resinación con pasta 10 frente a los estimulados con $\mathrm{H}_{2} \mathrm{SO}_{4}$ al $40 \%$. En la Fotografía 2.b, se aprecian árboles resinados mediante el sistema de pica de corteza sobre cara ancha frente a otros testigos sin resinar.

Aunque no se ha podido encontrar ninguna publicación o informe sobre la experiencia, en un documento de 1970, se resume brevemente el estado de la experiencia que, según se aprecia en la Tabla 4, alcanzó una media de producción de $3,1 \mathrm{~kg}$ a los 3 años en la parcela estimulada con ácido líquido y, en la parcela de 1969 (pasta frente a frente a $\mathrm{H}_{2} \mathrm{SO}_{4}$ al $40 \%$ liquido), hubo diferencias poco significativas al tener quizás una sola entalladura.

De los documentos estudiados, nada se desprende sobre el control de pérdida de crecimiento en madera ni tampoco se ha encontrado ninguna publicación de los resultados; no obstante, D. Miguel de Gaviña Mugica, responsable de la sección de resinas y productos derivados del IFIE en
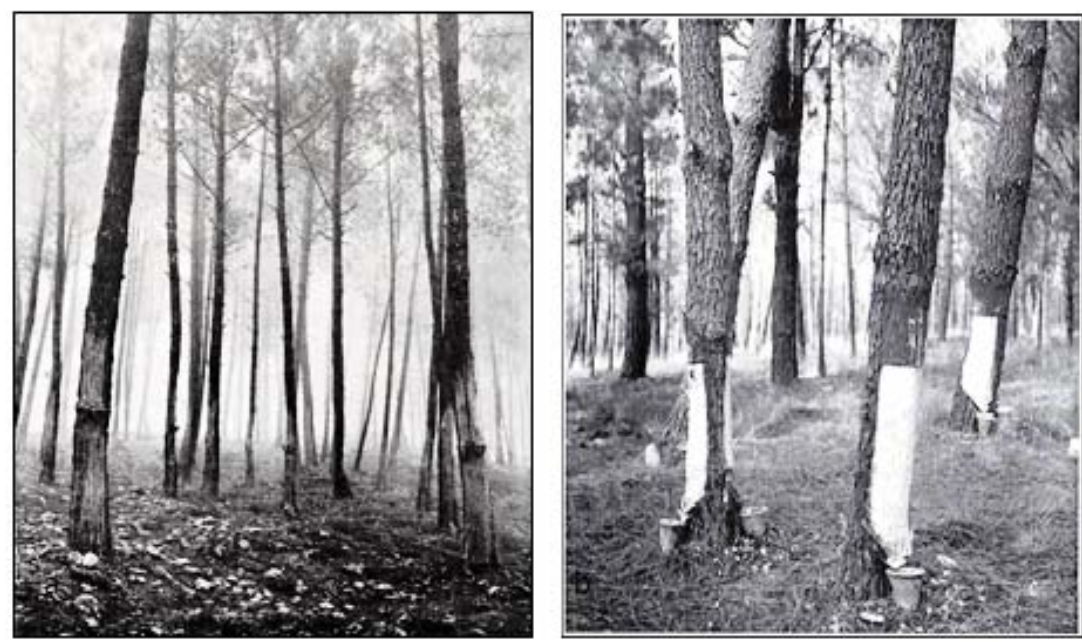

Fotografía 2.- a. Monte en resinación en Entrimo (Ourense). Fuente: fototeca forestal. INIA. b. Ensayo de extracción de resina por el sistema de pica de corteza con cara ancha. Localización probable: monte Seixos Albos (Tui, Pontevedra). Fuente: biblioteca CIF de Lourizán

\begin{tabular}{|c|c|c|c|}
\hline & $\begin{array}{c}\text { Parcela de } \\
1967\end{array}$ & \multicolumn{2}{|c|}{$\begin{array}{l}\text { Parcela } \\
\text { de } 1969\end{array}$} \\
\hline Tipo de estimulante & Ácido & Ácido & Pasta \\
\hline $\begin{array}{c}\text { Entalladura } 1^{\circ} . \\
\text { Año } 1967\end{array}$ & 2,756 & & \\
\hline $\begin{array}{c}\text { Entalladura } 2^{\circ} \text {. } \\
\text { Año } 1968\end{array}$ & 2,931 & & \\
\hline $\begin{array}{c}\text { Entalladura } 3^{\circ} \text {. } \\
\text { Año } 1969\end{array}$ & 3,620 & & \\
\hline $\begin{array}{c}\text { Entalladura } 1^{\circ} \text {. } \\
\text { Año } 1969\end{array}$ & - & 2,388 & 2,037 \\
\hline Producción media en $\mathrm{Kg}$ : & 3,102 & & \\
\hline
\end{tabular}

Tabla 4.- Datos a los 3 años de la experiencia de resinación mediante el sistema de pica con corteza con estimulante líquido y pasta en Seixos Albos (Tui, Pontevedra) 
octubre de 1966, en carta destinada al Jefe del Distrito de Pontevedra D. Ricardo García-Borregón, estima que las posibles pérdidas serian inferiores al $19 \%$ del crecimiento corriente anual a partir del momento en que comienza la resinación, siempre que esta se lleve a cabo como máximo en los 5 años precedentes al apeo por corta final.

Este tema, de capital importancia para el caso de una resinación complementaria al aprovechamiento maderero, sí fue algo más estudiado en Portugal, donde se iniciaron ensayos en la década de los cincuenta para determinar el decrecimiento del diámetro normal en la Mata Nacional de Leiria (Gomes,1954). Últimamente, y basado en una hipótesis teórica, Guardia, en el $1^{\circ}$ Simposio de Aprovechamiento de Resinas Naturales celebrado en Segovia, considera que la reducción anual en la producción de madera es del 10\% (Guardia, 1998), tomando como referencia el estudio de Figueiredo sobre el análisis de árboles de Pinus elliottii, donde la reducción del aumento de volumen sin corteza fue del $14,99 \%$ y el de la altura total, del 12,02 \% (Figueiredo, 1991). No obstante, lo que sí ha quedado demostrado es que, cuanto mayor es la producción de miera, menores son los crecimientos diamétricos de los árboles (Palma, 2007).

La última experiencia de la que se tiene conocimiento en Galicia es la que propone el IFIE en 1970, titulada: "Estudio de la resinación en Galicia mediante el sistema de pica de corteza con estimulantes sólidos sobre caras anchas" (IFIE, 1970), donde se pretendía realizar un estudio sistemático de la resinación a base del sistema de pica de corteza con estimulante sólido ejecutado sobre caras de anchura superior a la normal en España, establecida en $12 \mathrm{~cm}$, proponiendo el estudio contrastar, durante 5 años (19711975), la resinación sobre caras de $20 \mathrm{~cm}, 16 \mathrm{~cm}$ y $12 \mathrm{~cm}$ (testigos) y distintos sistemas de recogida en función de la anchura y de la compatibilidad con el aprovechamiento maderero, realizando, así mismo, un control de la pérdida de crecimiento debido a la resinación.

Prácticamente nada se ha encontrado de las que quizás fueron las últimas experiencias, a excepción de la propuesta del año 1970 para realizar la experiencia en los montes de Alto de Sobrada, de la parroquia de Sobrada, y en el monte Cabritas, de la parroquia de Currás, ambos en el ayuntamiento de Tomiño.

\section{Discusión}

En primer lugar, lo más destacable de esta revisión son las numerosas experiencias y ensayos de resinación que se realizaron en Galicia entre la década de los 50 y los 60 y lo poco conocidos que son, tanto en el ámbito forestal gallego como en el nacional. Aunque estos ensayos fueron numerosos, es verdad que estuvieron bastante localizados en la provincia de Pontevedra, quedando fuera otras localizaciones geográficas, como el centro y sur de la provincia de Ourense que, por su climatología y geografía (temperatura, precipitaciones, humedad relativa, exposición, etc.), podrían tener más interés para la producción de miera; todo ello quizás motivado por la falta de masas de Pinus pinaster Ait. de dimensiones suficientes.
Es necesario resaltar que los documento estudiados, o adolecen de un tratamiento estadístico de los resultados del diseño experimental, o no se ha encontrado, por lo que los valores obtenidos en los diferentes ensayos tienen una validez relativa.

Es destacable, entre todas las experiencias, la realizada por la Diputación de Pontevedra en el Morrazo (Pontevedra) por su magnitud, tanto en el tiempo, 5 años, como en el número de pinos resinados, 22000 pies. Esta importante experiencia y las otras realizadas, que en su conjunto superaron de media los $2 \mathrm{~kg}$ lárbol, son representativas de las posibles producciones que podrían obtenerse en Galicia por árbol y campaña de resinación; valores que se consideran dentro del grado de la productividad del Pinus pinaster, que oscila entre 2 y $3 \mathrm{~kg} / a$ rbol de media (Picardo, 2013).

Otra cuestión destacable es la preocupación, desde los primeros ensayos, del efecto que la resinación tendría en la pérdida de crecimiento de los árboles y, en definitiva, en el volumen maderable, aunque poco o nada se ha encontrado de la perturbación que el método de resinación propuesto (2 o 5 años antes de la corta) podría tener también en la calidad de la madera. Hoy, es un hecho constatable que la modalidad de resinación a vida es difícilmente compatible con el aprovechamiento de la madera para sierra; sin embargo, la modalidad a muerte permitiría la consecución de madera de calidad y la obtención de resina como producto secundario (Anastácio \& Carvalho).

En la dos décadas de ensayos que se realizaron en Galicia, los sistemas de resinación empleados fueron evolucionando hasta el sistema de pica de corteza con estimulación con pasta química, que, al final, se llegó a implantar en el resto de la Península Ibérica hasta la actualidad, y que no se consolidó en Galicia por la falta de tradición resinera y, también, por la llegada de la crisis en los precios de la miera. Circunstancia de gran trascendencia, toda vez que este método implica una serie de ventajas sobre los otros sistemas: la compatibilidad del aprovechamiento resinero y maderero, la mayor productividad, y la mayor facilidad de aprendizaje (Hernández, 2006).

\section{Conclusiones}

De la documentación estudiada, se concluye que el antiguo IFIE mantuvo líneas de experiencias durante, al menos, dos décadas en Galicia, con el fin de encontrar un método de resinación complementario al aprovechamiento principal o maderero, limitándolo, como máximo, a los dos o cinco años previos a la corta final, sin llegar a proponer la resinación como aprovechamiento principal.

Estas experiencias, aparte de probar diferentes sistemas de resinación, apuntaron soluciones a los factores naturales que más pueden influir en la producción de resinas en Galicia, como son los climáticos (precipitaciones, temperaturas, etc.) y los selvícolas (densidad, diámetro normal, biomasa, etc.), y dejaron pendiente el estudio de otros factores naturales de gran influencia en la producción, 
como son los geográficos (latitud, altitud y exposición) y los biológicos (subespecies, procedencias y ecotipo).

La mayor parte de las experiencias, por la época en que se realizaron, poco aportan ya de interés sobre los factores tecnológicos, como son los sistemas de resinación, la intensidad de la misma, la época, los estimulantes, etc.

Finalmente, y aunque no se pueden extraer conclusiones concretas de las producciones obtenidas, de estos estudios si se puede obtener orientaciones para futuras investigaciones que permitan, primeramente, decidir sobre la viabilidad de la resinación en Galicia, teniendo en cuenta, tanto la cantidad como la calidad de la miera que pueda producirse, así como el posterior desarrollo de métodos y sistemas de resinación adaptados a las características del medio forestal gallego.

Agradecimientos A D. Juan Carlos Álvarez, presidente de la Mesa de la Resina en Castilla y León y de la Asociación Nacional para la Defensa y Vertebración del Sector Resinero, por la donación de la documentación inédita del IFIE que poseía; al Centro de Investigación Forestal de Lourizán de la Xunta de Galicia, esencial para poder realizar este artículo.

\section{Bibliografía}

Anastácio, D. \& J. Carvalho (2008). Sector dos resinosos em Portugal. Evolução e análise. D.G.R.F.

Blanco, S. (2011). "La resinación por el método de pica de corteza ascendente. Estudio de tiempos, rendimientos y costes en distintos montes de la provincia de Segovia". Disponible en http://oa.upm.es/7099/

Calderón, A. (2009). "Industrias y productos forestales. Cuadernos de dasonomía". Serie Didáctica No 14, Departamento de Producción Agropecuaria Facultad de Ciencias Agrarias. Universidad Nacional de Cuyo.

Figueiredo, A. (1991). Influência da Resinagem no Crescimento de Pinus elliottii var. elliottii e sua Avaliação Econômica. Universidade Federal do Paraná, Doutoramento.

García-Borregón R. (1966). Carta del ingeniero Jefe del Distrito Forestal de Pontevedra a Miguel Gaviña Mújica sobre el "Informe influencia de la resinación en relación con el aprovechamiento de la madera realizado por la Sección de Resinas del IFIE. Documento inédito de la biblioteca del CIF de Lourizán.

Gomes, A. (1954). Sobre a influência da resinagem no crescimento do Pinheiro bravo I - Crescimento em diâmetro. Ministério da Economia, DGSFA. Estudos e Informação n $43-E 3$, Lisboa.

Guardia, N. (1998). "Repercusiones socioeconómicas de la resinación en montes privados". En 1er Simposio de Aprovechamiento de Resinas Naturales. Segovia, 5-7 de febrero.

Hernández, L. (2006). El antiguo oficio de resinero. Ministerio de Agricultura, Pesca y Alimentación. 2006. Hoja divulgativas $n^{\circ} 2116.31$ págs. ISBN, 84-491-0687-7.
IFIE. (1962). "Informe sobre las experiencias de producción de miera por los nuevos sistemas de resinación", por la Sección de resinas y productos derivados del IFIE., n. 7, p.177-211.

IFIE. (1965). "Informe sobre las experiencias de producción de miera por los nuevos sistemas de resinación", por la Sección de resinas y productos derivados del IFIE., n. 7, p.177-211.

IFIE. Sección de resinas y productos derivados. (1967). "Estudio relativo a la influencia que ejerce la resinación, por el sistema de pica de corteza con estimulación, en el crecimiento maderable". Documento inédito del IFIEbiblioteca Lourizán.

IFIE. Sección de resinas y productos derivados. (1970). "Propuesta general sobre el estudio de la resinación en Galicia mediante el sistema de pica de corteza con estimulante sólidos, sobre caras anchas". Documento inédito del IFIE- biblioteca Lourizán.

Nájera, F. \& R. Boulet. (1961). "Informe sobre la resinación de los pinares de Galicia y condiciones generales que se proponen para la concesión de este aprovechamiento". Documento inédito del IFIE-biblioteca Lourizán.

Nájera, F. (1957). "Informe sobre la posible resinación de los pinares de Galicia y estudio de las bases generales que deberá cumplir este aprovechamiento". Documento inédito de la biblioteca del CIF de Lourizán.

Nájera, F.; Rifé, P.; Carrascosa, T. \& E. Fajó. (1956). "Experiencias de producción de miera. Sección de resinas y productos derivados". Anales del Instituto Forestal de Investigaciones y Experiencias, n.1, p. 103-112.

Nájera, F.; Rifé, P.; Carrascosa, T. \& E. Fajó (1957). "Técnicas Modernas en la resinación de los pinos españoles. Sistema de espina de pescado con cepillo Mazek". Sección de resinas y productos derivados. Anales del Instituto Forestal de Investigaciones y Experiencias, n.2, p. $125-141$

Palma, A., (2007). Capacidade produtiva de resina do pinheiro bravo: Breve panorâmica do sector resineiro em Portugal, Trabalho apresentado como dissertação original para efeitos de acesso à categoria de Investigador Auxiliar, Oeiras: Instituto Nacional de Recursos Biológicos - Estação Florestal Nacional.

Picardo, A. (2013). "La resinación en España y en el mundo en 2013: Situación y perspectivas". Actas del II Simposio Internacional. de Resinas. Naturales. II Coca Segovia 16 al 18 de abril de 2013. Disponible en http://4.interregsudoe.eu/contenido-dinamico/libreria-ficheros/082459767B8C-2E71-B891-FDAEB7077CF8.pdf

Pinillos, F.; Picardo, A.; Allué-Andrade, M.; Soria, E. \& A. Sanz. (2004). La resina: Herramienta de conservación de nuestros pinares, basada en el contenido del "Informe diagnóstico y propuestas de actuación en el sector resinero".

Radich, M. (1995). "O saber da resinagem em Portugal". Ler História 27/28:177-199. 
Rodríguez, R.; Serrada, R.; Lucas, J.; Alejano, R.; Del Río, M.; Torres, E. \& A. Cantero. (2008). "Selvicultura de Pinus pinaster Ait. subsp. mesogeensis Fiéis \& Gaussen". En: Serrada, R.; Montero, G.; Reque, J. (Eds.), Compendio de selvicultura aplicada en España. INIA, 399-430.

Santos, C. (2013). Resinagem em Portugal. Situação Atual e Perspetivas Futuras. Disponible en http://www.sustforest.eu/sites/www.sust-forest.eu/files/cristina_santos.pdf
Serrada, R. (2004). Apuntes de selvicultura. Fundación Conde del valle de Salazar. E.U.I.T. Forestal, Madrid.

Solís, W. (1968). "Experiencias de resinación con el sistema de espina de pescado con el cepillo Mazek sobre Pinus pinea". Comunicaciones del IFIE n. 2, p.10. 